Preference is given to letters commenting on contributions published recently in the JRSM. They should not exceed 300 words and should be typed double spaced

\section{Surviving a viva}

Mr Cascarini and Professor Lowe's advice to viva candidates (October $2004 J R S M^{1}$ ) will have struck a chord with many past examinees and certainly did so in this examiner. Correctly the authors summarize the essentials for a passpossess a thorough knowledge and convey this to the examiners. For most serious candidates an oral examination comes down to good communication skills in convincing revered examiners of a safe understanding of the subject.

I suspect anxiety or examination nerves substantially degrade the performance of many candidates and steps can reasonably be taken to overcome these. After all, there is a huge difference between dealing with colleagues and relatives and communicating with an examiner who appears to hold all the cards. My advice is to use a modest dose of a beta-blocker such as propranolol. These agents remove the debilitating effects of the adrenalated state without lessening the pressure to perform well. Experience from lecturing in stressful situations has demonstrated that a degree of betablockade allows one to handle aggressive questioning with an apparent time advantage in understanding the point of the question and arranging a suitable reply even before the questioner has finished.

Educationalists question the validity of the oral examination process because of variables such as examination nerves. Could anyone object to minimizing this variable by pharmacological means? Is it unfair? I think not. Be careful, however, of pharmacological interactions immediately after the examination when comparing notes in the bar.

\section{Ian Fraser}

Warwick Hospital, Warwick CV34 5BW, UK

\section{REFERENCE}

1 Cascarini L, Lowe DG. Surviving a viva: a guide for candidates. J R Soc Med 2004;97:498-500

\section{Research funding in complementary medicine}

Professor Ernst (October $2004 J R S M^{1}$ ) believes the UK Department of Health to be misguided in funding isolated postdoctorate fellowships in complementary medicine rather than 'centres of excellence' such as his own in Exeter. In my opinion, individual funding is the better route for people who wish to do practical research. The metaanalyses that represent a large part of the output of the instances such studies ought to be conducted as preliminaries to new work in the laboratory or clinic. Professor Ernst's centre has also reported randomized trials, but there are dangers in conducting these from a centre dealing with 'complementary' techniques in general rather than in particular. Sometimes these have come under severe criticism from complementary practitioners. From my specialist knowledge of acupuncture, I have expressed grave doubts about the design and conclusions from one of the trials published from the Exeter unit. ${ }^{2}$ In terms of quality, the Department of Health may have chosen the best course.

\section{Bo-Ying Ma}

Xinglin Postgraduate College of Chinese Medicine (UK), London, UK E-mail: collegexpct@tiscali.co.uk

\section{REFERENCES}

1 Ernst E. Research capacity in complementary medicine. $J$ S Soc Med 2004;97:504-5

2 Chen HY, Ma BY, Grant A. Research funds for complementary medicine. Lancet 2001;357:1982

\section{Assessment}

Professor Baskerville (November $2004 J R S M^{1}$ ) describes how his father became caught up in a system designed to meet the needs of people providing care and not the needs of the patients. The saga ended in a casualty department when a young doctor conducted a cognitive assessment. The abbreviated mental test is a screening test for dementia and must be done carefully. A study conducted in a district general hospital indicated that this does not happen. ${ }^{2}$ Doctors carrying out the test often do not explain what cognitive tests are about or ask the patient's permission to administer the test or ensure the patient's privacy. They often do not realize that cognitive tests may be impossible to perform on a patient with dysphasia, hearing impairment or visual impairment - which should be ruled out first. They often verbally or non-verbally prompt the patient and sometimes they press too hard on items that the patient finds difficult, precipitating a catastrophic reaction. They often accept patients' excuses as to why they do not know the answer-not realizing that patients with dementia effectively hide behind these excuses. Sometimes they allow the patient to make multiple attempts to answer a question. They seldom realize that the test score must be interpreted in the context of the history and examination and other tests: dementia is not to be diagnosed on the basis of a single low score. Finally they do not realize that the test can be distressing and humiliating and do not always reassure the patient at the end. Why do they make all these errors? Because nobody has shown them how to do it correctly. If we gave house officers a colonoscope and told them to screen patients for colorectal cancer every one would be 
appalled. But we let them screen patients for dementia without another thought.

\section{Kieran Walsh}

8 Elmhurst Villas, Cheltenham Road, London SE15 3AE, UK

E-mail: bmjlearning.com

\section{REFERENCES}

1 Baskerville A. Assessment. J R Soc Med 2004;97:547-8

2 Walsh K, Gupta I, Mullane D, Chan C. Administration of the minimental state examination by junior doctors at a district general hospital: an observational study [Abstract]. Ir J Med Sci 2002;170:106

\section{Prevention of falls in hospital inpatients}

The recommendations of Dr Vassallo and his colleagues (June $2004 \mathrm{JRSM}^{1}$ ) for prevention of falls in patients with unsafe gait, by simple risk stratification and targeting, seem a notably practical option. They identify confusion and previous falls as the main predictors. It is important to highlight that most of the patients in the study were elderly (mean age 82 years).

Falls are also very troublesome in post-acute rehabilitation in younger patients. The Alderbourne Neurorehabilitation Unit treats a younger age group of patients with traumatic brain injury, multiple sclerosis, strokes, and non-traumatic spinal cord injury. The average age in May 2004 was 56 . We audit all the incident forms pertaining to falls on the unit and a pattern similar to that observed by Vassallo et al. is emerging.

During the eighteen months January 2003 to June 2004 there were 34 falls recorded among inpatients admitted for rehabilitation. 19 (55\%) were isolated incidents but the other 15 were recurrent falls ( 6 patients with an average of 2.5 falls each). All of these fallers had cognitive impairment. Whilst most of these falls only resulted in minor injury, 2 resulted in trauma sufficient to affect the ability to participate in rehabilitation and increased the length of stay.

Whilst a history of previous falls is a predictor of potential risk of future falls, cognitive impairment is the other predictor that requires highlighting. Low scores on three items of the admission Functional Independence Measure (a standardized outcome measure regularly used on neurological rehabilitation units) - namely, comprehension, memory and safety awareness - coupled with a history of falls, should assist in the risk assessment process for these patients.

Having identified patients at high risk of falls the interventions to prevent further falls that have been reviewed include alert bracelets, ${ }^{2}$ attention to flooring, ${ }^{3}$ and corridor lighting, additional exercises as well as a multidisciplinary multiple intervention programme including the use of hip protectors. Vassallo et al. state that the 'arguments for a multifactorial intervention remain weak'.
However in a randomized controlled trial Haines et al. ${ }^{4}$ showed a $30 \%$ reduction in falls using a targeted multiple interventions falls prevention programme. Chang et al. ${ }^{5}$ likewise concluded that such programmes are effective.

A Nair

H Parr

H Whitelock

R S Hanspal

Alderbourne Rehabilitation Unit, Hillingdon Hospital, Uxbridge UB8 3NN, UK E-mail: ajoynair@aol.com

\section{REFERENCES}

1 Vassallo M, Vignaraja R, Sharma JC, Briggs K, Allen S. Predictors for falls among hospital inpatients with impaired mobility. $J$ R Soc Med 2004;97:266-9

2 Mayo, N, Gloutney L, Levy R. A randomised trial of identification bracelets to prevent falls among patients in a rehabilitation hospital. Arch Phys Med Rehabil 1994;75:1302-8

3 Donald I, Shuttleworth H. Preventing falls on an elderly care rehabilitation ward. Clin Rehabil 2000; 14:178-85

4 Haines TP, Bennell KL, Osborne RH, Hill KD. Effectiveness of a targeted falls prevention program in a sub-acute hospital setting - a randomised controlled trial. BMJ 2004;328:676-9

5 Chang JT, Morton SC, Rubenstein LZ, et al. Interventions for the prevention of falls in older adults: systematic review and meta-analysis of randomised clinical trials. BMJ 2004;328:680-3

\section{Medical acronyms}

In this acronymous age, articles are often difficult to read, even incomprehensible, because of the numerous abbreviations. Professor Cheng's solution (November 2004 JRSM $^{1}$ ) strikes me as impractical. However, a few journals have a full list of acronyms spelt out with their meanings, at the end of or the beginning of articles. This I find invaluable and commend for your consideration, Sir, for our journal.

\section{J N Blau}

City of London Migraine Clinic, 22 Charterhouse Square, London EC1M 6DX, UK

\section{REFERENCE}

1 Cheng TO. Medical abbreviations. J R Soc Med 2004;97:556

\section{Palliative care in community hospitals}

Professor Payne and her colleagues (September 2004 $J R S M^{1}$ ) conclude that community hospitals represent an under-used resource for palliative care. I surveyed the use of an urban community hospital for each of the years 1987 2004 when I worked in my previous practice of 8000 patients in Haydock, St Helens. During these 17 years we cared for 249 patients who died of malignant disease when at least part of the terminal phase was spent in the community. 
The place of death, and numbers (percentage) of these patients was:

General/tertiary hospital

Own home

Nursing/residential home

Community hospital

Hospices

Although on average only 3 patients died in the community hospital each year, this was a particularly useful option for those without a family able to care for them at home. It also made an important contribution to our aim of keeping as many patients as possible, when this was desired and appropriate, under our care at death. We achieved this for $74 \%$ of patients - a percentage that remained constant. This was probably helped by the out-of-hours service being asked to pass any calls for such patients back to a general practitioner from the practice.

One relative has stated his definition of good palliative care: 'the essential concept is that the doctor (or at least the practice) will stay firmly with the patient and relative at their time of need and not desert them'. ${ }^{2}$ Community hospitals have an essential role in the provision of this and other aspects of primary palliative care, which should be developed further. ${ }^{3}$

John Holden

Garswood Surgery, Station Road, Garswood WN4 OSD, UK

\section{REFERENCES}

1 Payne S, Kerr C, Hawker S, et al. Community hospitals: an underrecognized resource for palliative care. $J$ R Soc Med 2004;97:428-31

2 Brewin T. Personal view-deserted. BMJ 2001;322:117

3 Murray SA, Boyd K, Sheikh A, Thomas K, Higginson I. Developing primary palliative care. BMJ 2004;329:1056-7

\section{Needle-stick injuries in the National Health Service}

Elmiyeh and co-workers (July $2004 J R S M^{1}$ ) report a culture of silence on needle-stick injuries. As they indicate, clinicians do not easily change their practice. $^{2}$
The case for post-exposure prophylaxis is well grounded ${ }^{3}$ though not yet (understandably) proven by a randomized controlled study. In the UK, the needle-stick injury needs to be reported in order to access such treatment, via occupational health or emergency services. However, there are other less tangible reasons for reporting. Criminal liability has been established for actions taken by a person who knew himself to be HIV-positive. ${ }^{4}$ What about the liability of doctors who know they have sustained a potentially infecting needle-stick injury? The Selby train crash showed that the driver who knew he was too tired to drive was liable for the consequences of falling asleep. $^{5}$ Therefore there is a risk of liability for actions while hazardously infected, through an act of omission. This can only be determined by a legal test case, which I would not wish to be part of.

The final benefit is the certainty that comes from appropriate and conclusive infectious status testing. It was the above factors rather than the system-wide implications that I found most motivating - plus, as stated by Elmiyeh et al. ${ }^{1}$ the possibility of invalidating insurance cover - when several months ago I reported my own needle-stick injury from a high-risk case.

\section{David Wallace}

Craniofacial Department,

Chelsea and Westminster Hospital,

London SW10 9NH, UK

E-mail: david.wallace@nhs.net

\section{REFERENCES}

1 Elmiyeh B, Whitaker IS, James MJ, et al. Needle-stick injuries in the National Health Service: a culture of silence. J R.Soc Med 2004;97:326-7.

2 Foy R, Walker A, Penney G. Barriers to clinical guidelines: the need for concerted action. Br J Clin Gov 2001;6:166-74.

3 van der Ende ME, Regez RM, Schreij G, et al. Post-exposure prophylaxis. Int J STD AIDS 2002;13 Suppl. 2:30-4.

4 Pook S. HIV refugee who infected lovers may die in jail. Daily Telegraph 15 October 2003.

5 Stokes P. Selby train crash driver is jailed for five years. Daily Telegraph. 12 January 2002. 